\title{
Исследование длинноволновых инфракрасных спектров отражения моно- и поликристаллов SmS в области гомогенности
}

\author{
(C) Ю.В. Улашкевич1,2, В.В. Каминский ${ }^{2}$ ฯ, М.В. Романова ${ }^{2}$, Н.В. Шаренкова ${ }^{2}$ \\ ${ }^{1}$ Санкт-Петербургский государственный технологический институт (технический университет), \\ 190013 Санкт-Петербург, Россия \\ 2 Физико-технический институт им. А.Ф. Иофффе Российской академии наук, \\ 194021 Санкт-Петербург, Россия \\ I E-mail: vladimir.kaminski@mail.ioffe.ru
}

(Получена 23 мая 2017 г. Принята к печати 31 мая 2017 г.)

\begin{abstract}
Зарегистрированы и проанализированы спектры отражения в дальней и средней инфракрасных областях образцов $\mathrm{Sm}_{1+x} \mathrm{~S}(x=0-0.17)$, а также их электрические и структурные параметры при температуре $T=300 \mathrm{~K}$. Показано, что степень ионности связи в $\mathrm{SmS}$ уменьшается по мере уменьшения величины области когерентного рассеяния рентгеновского излучения, увеличения концентрации донорных примесей и, как следствие, концентрации электронов проводимости. В моно- и поликристаллах $\mathrm{SmS}$ стехиометрического состава электропроводность может с точностью $10 \%$ определяться из инфракрасных спектров отражения. При отклонении от стехиометрии повышенная дефектность образцов не дает возможности определять электропроводность.
\end{abstract}

DOI: $10.21883 /$ FTP.2018.02.45441.8656

\section{1. Введение}

Халькогениды самария используются в тензодатчиках, являются перспективными материалами для термогенераторов и газоанализаторов [1-3]. Поэтому электрические, тепловые и магнитные свойства данных соединений интенсивно исследуются в течение полувека. Результаты этих исследований наиболее подробно изложены в монографии [4]. При исследованиях термовольтаического эффекта в гетероструктурах необходимо получить данные об электрических параметрах образца в приповерхностном слое [5]. Поэтому оптические методы, в которых рассматриваются частотные зависимости поглощения и отражения полупроводников, являются хорошим альтернативным решением для вычисления электрофизических параметров этих материалов [6]. Однако в [4] было отмечено, что исследований оптических свойств $\mathrm{SmS}$ имеется очень мало. По-видимому, ранее это было связано со сложностью изготовления совершенных моно- и поликристаллов халькогенидов самария. Лишь по мере разработки технологии изготовления таких материалов, в частности $\mathrm{SmS}$, стали возможны их оптические исследования в инфракрасной (ИК) области, требующие наиболее совершенных кристаллов. Так, подробное исследование спектров отражения монокристалла сульфида самария в длинноволновом ИК диапазоне (FIR) [7] позволило на основании модели Друде-Лоренца, учитывающей вклад колебаний фононов и плазмы свободных электронов, вычислить ряд электрофизических параметров этого кристалла и показать их хорошую корреляцию с величинами, полученными из электрических измерений. Аналогичные закономерности были получены при рассмотрении длинноволнового ИК спектра отражения поликристаллического образца $\mathrm{SmS}$ стехиометрического состава [5]. Особенности спектров отражения в средней ИК области (MIR) для поликри- сталлических образцов моносульфида самария с составами, находящимися в области его гомогенности, были исследованы в работе [8]. На основании этих исследований были выявлены смещение и мультиплетное расщепление уровня ${ }^{7} F_{2}$ и определены их количественные значения.

В плане продолжения исследований, начатых в [5], в настоящей работе проведено исследование спектров отражения поликристаллов моносульфида самария с составами, находящимися в области его гомогенности, сравнение их со спектрами монокристаллов и нахождение корреляционных зависимостей между оптическими и электрическими параметрами образцов. С прикладной точки зрения это важно для оценки электрических параметров различных областей гетероструктур на основе $\mathrm{SmS}$, применяемых для исследования термовольтаического эффекта.

\section{2. Образцы и методика эксперимента}

Для проведения спектральных измерений были использованы поликристаллические образцы моносульфида самария с составами, находящимися в области его гомогенности: $\mathrm{Sm}_{1+x} \mathrm{~S}(x=0-0.17)$. Синтез образцов описан в работе [9]. В основном образцы имели размеры $3 \times 5 \times 20$ мм, а в тех случаях, когда размеры были нестандартными, использовались диафрагмы подходящей формы. Одновременно для сравнения были исследованы два монокристалла $\mathrm{SmS}$.

Регистрация спектров отражения $R(v)$ проводилась при комнатной температуре на инфракрасном фурьеспектрометре IFS-113v фирмы Bruker в диапазоне частот $v=20-4000 \mathrm{~cm}^{-1}$ с разрешением $2 \mathrm{~cm}^{-1}$. Использовались майларовые светоделители различной толщины, а также светоделитель $\mathrm{KBr}$. В качестве источников излучения для диапазонов FIR и MIR применялись дейтериевая 
Таблица 1. Структурные и электрические характеристики образцов

\begin{tabular}{|c|c|c|c|c|c|c|c|c|c|}
\hline $\begin{array}{l}\text { Номер } \\
\text { образца }\end{array}$ & $x$ & $\begin{array}{l}a, \\
\AA\end{array}$ & $\begin{array}{c}\text { ОКР, } \\
\AA\end{array}$ & $\begin{array}{c}N_{i}, \\
10^{20} \mathrm{~cm}^{-3}\end{array}$ & $\begin{array}{c}\sigma_{\mathrm{el}}, \\
\mathrm{OM}^{-1} \cdot \mathrm{cm}^{-1}\end{array}$ & $\begin{array}{c}\sigma_{\mathrm{opt}}, \\
\mathrm{OM}^{-1} \cdot \mathrm{cm}^{-1}\end{array}$ & $\begin{array}{c}n, \\
10^{18} \mathrm{~cm}^{-3}\end{array}$ & $m_{c}^{*}$ & $e_{s}^{*}$ \\
\hline 1 & $0.00^{*}$ & 5.968 & 2500 & 1.78 & 37 & 36.4 & 5.2 & 0.16 & 0.85 \\
\hline 2 & $0.00^{*}$ & - & - & - & - & 17.3 & - & - & 0.92 \\
\hline 3 & 0 & 5.9675 & 550 & 11.80 & 19.6 & 18.2 & 6.04 & 0.91 & 0.62 \\
\hline 4 & 0.02 & 5.9687 & 750 & 8.04 & 44.6 & 11.2 & 34 & 11.31 & 0.21 \\
\hline 5 & 0.02 & - & 750 & - & 105 & 8.1 & - & - & 0.36 \\
\hline 6 & 0.04 & 5.9698 & 700 & 8.56 & 17.8 & 11.2 & 21.5 & 5.83 & 0.33 \\
\hline 7 & 0.04 & - & - & - & 104 & - & 34 & - & - \\
\hline 8 & 0.05 & - & - & - & 222 & 14.0 & 40 & 14.57 & 0.08 \\
\hline 9 & 0.05 & - & - & - & 52.6 & 41.2 & 21.7 & 1.88 & 0.37 \\
\hline 10 & 0.06 & 5.9725 & 570 & 11.2 & 12.4 & 7.8 & - & - & 0.19 \\
\hline 11 & 0.06 & - & - & - & 13 & 12.7 & - & - & 0.74 \\
\hline 12 & 0.07 & 5.9665 & 600 & 10.7 & 33.9 & - & 46 & - & - \\
\hline 13 & 0.08 & 5.9650 & 550 & 11.80 & 84.7 & 15.0 & 16.2 & 3.44 & 0.36 \\
\hline 14 & 0.08 & - & - & - & 119 & - & 34 & - & - \\
\hline 15 & 0.1 & - & - & - & - & 5.3 & - & - & 0.15 \\
\hline 16 & 0.1 & - & - & - & 95.2 & 19.0 & - & - & 0.50 \\
\hline 17 & 0.15 & - & - & - & 68.5 & 33.5 & - & - & - \\
\hline 18 & 0.16 & - & - & - & 267 & 9.1 & 35.8 & 13.7 & - \\
\hline 19 & 0.17 & 5.9660 & 500 & 13.19 & 16.3 & 15.4 & 34 & 12.6 & - \\
\hline 20 & 0.17 & - & - & - & 435 & 69.8 & - & - & - \\
\hline
\end{tabular}

Примечание. * - монокристаллический образец.

лампа и глобар соответственно. Спектры измерялись с помощью приставки зеркального отражения фирмы Bruker, угол падения луча на образец не превышал $8^{\circ}$. Для каждого образца проводилось несколько измерений, спектры усреднялись и „сшивались“ на стыке диапазонов FIR и MIR. Непосредственно перед спектральными измерениями образец подвергался химическому травлению, поскольку механическая полировка переводит полупроводниковый $\mathrm{SmS}$ в металлическое состояние.

Измерения удельной электропроводности $\sigma_{\mathrm{el}}$ кристаллов были проведены стандартным четырехзондовым методом при постоянном токе. Концентрация электронов $n$ измерена с помощью эффекта Холла. Рентгеноструктурный анализ (РСА) образцов был проведен дифрактометрическим методом $(\theta-2 \theta$-сканирование) на рентгеновском дифрактометре ДРОН-2 в $K_{\alpha}$-излучении медного анода. Величина области когерентного рассеяния рентгеновского излучения ОКР определялась методом аппроксимации из величин истинного уширения дифрактометрических отражений 200 и 111 по формуле Селякова-Шеррера $[10,11]$. Параметр кристаллической решетки $a$ получен усреднением вычисленных значений в интервале углов $2 \theta$ от 90 до $160^{\circ}$. Все структурные характеристики исследованных образцов и измеренные их электрические параметры приведены в табл. 1.

\section{3. Результаты исследования}

Некоторые из зарегистрированных спектров отражения для монокристаллов $\mathrm{SmS}$ и поликристаллов различного стехиометрического состава в наиболее информативном диапазоне $20-700 \mathrm{~cm}^{-1}$ представлены на рис. 1. Можно видеть, что спектры на этом рисунке имеют однотипный характер: в низкочастотной области кривая отражения круто устремляется вверх, стремясь к полному отражению при $v \rightarrow 0$, в высокочастотной области отражение монотонно уменьшается. На крае измеренного диапазона, $4000 \mathrm{~cm}^{-1}$, отражение не превышает нескольких процентов. При волновом числе $v \approx 185 \mathrm{~cm}^{-1}$ наблюдается пик отражения, обусловленный колебаниями кристаллической решетки.

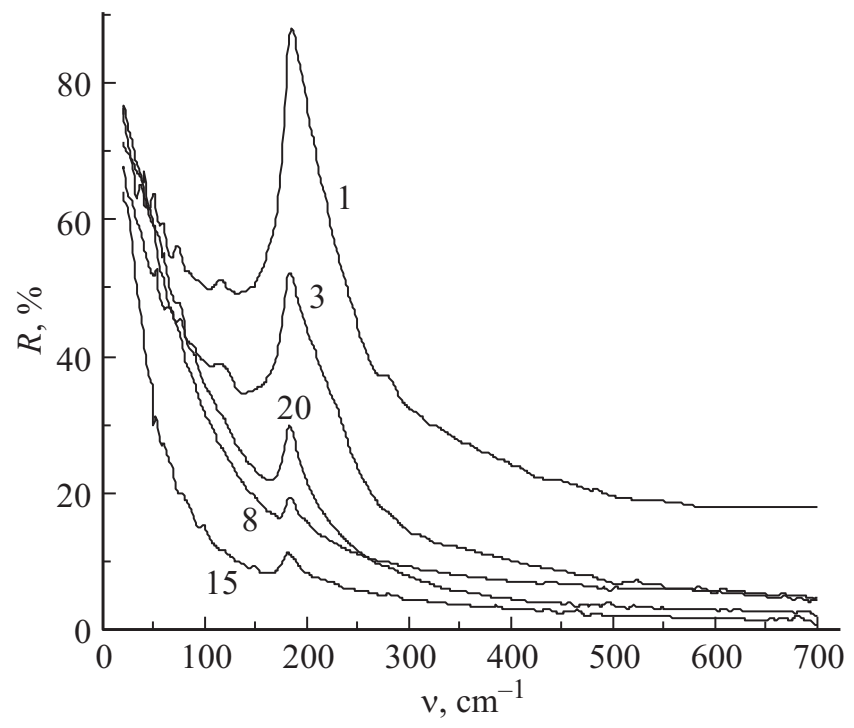

Рис. 1. Спектры отражения образцов $\mathrm{Sm}_{1+x} \mathrm{~S}$ : монокристаллического образца 1 и поликристаллов $3,8,15,20$ (номера образцов соответствуют табл. 1). 
Для образцов 7, 12 и 14 зарегистрированные спектры имеют схожие особенности (см. рис. 2) и вместе с тем кардинально отличаются от спектров на рис. 1. В [12] было отмечено, что при количестве $\mathrm{Sm}<50$ ат\% получаемый при синтезе материал всегда имеет две фазы, одна из которых представляет собой состав $\mathrm{Sm}_{3} \mathrm{~S}_{4}$. Более того, некоторая незначительная часть второй фазы может существовать и в области гомогенности. Для выяснения возможности искажения спектров $\mathrm{Sm}_{1+x} \mathrm{~S}$ за счет второй фазы в настоящей работе был зарегистрирован спектр отражения монокристалла $\mathrm{Sm}_{3} \mathrm{~S}_{4}$. Он также представлен на рис. 2, из которого видно, что спектры указанных выше образцов имеют некоторые характерные для $\mathrm{Sm}_{3} \mathrm{~S}_{4}$ особенности. Вместе с тем наблюдаются полосы, не свойственные этому соединению. Поэтому для ряда образцов был проведен рентгеноструктурный анализ их поверхности. Для образца 12 он показал, что поверхность лишь на $28 \%$ состоит из фазы $\mathrm{SmS}$, остальная часть содержит оксисульфид самария, $\mathrm{Sm}_{2} \mathrm{O}_{2} \mathrm{~S}$. Это же соединение было обнаружено на поверхности образца 10 в количестве $\sim 18 \%$. В остальных образцах примеси имеют лишь следовой характер на фоне основной фазы $\mathrm{SmS}$. Их спектры и представлены на рис. 1. Таким образом, хотя для образцов 7, 12 и 14 электрические параметры не выделяются на фоне остальных образцов (см. табл. 1), их спектры отражения искажены за счет наличия посторонних фаз. Такие образцы были исключены из дальнейших обсуждений и в статье рассматриваются только кристаллы со спектрами типа представленных на рис. 1.

При нормальном падении излучения на образец коэффициент отражения $R$ может быть выражен формулой [6]

$R(v)=\frac{1+\sqrt{\varepsilon_{1}(v)^{2}+\varepsilon_{2}(v)^{2}}-\sqrt{2\left[\varepsilon_{1}(v)+\sqrt{\varepsilon_{1}(v)^{2}+\varepsilon_{2}(v)^{2}}\right]}}{1+\sqrt{\varepsilon_{1}(v)^{2}+\varepsilon_{2}(v)^{2}}+\sqrt{2\left[\varepsilon_{1}(v)+\sqrt{\varepsilon_{1}(v)^{2}+\varepsilon_{2}(v)^{2}}\right]}}$,

где $\varepsilon_{1}(v)$ и $\varepsilon_{2}(v)$ - действительная и мнимая части диэлектрической проницаемости $\varepsilon(v)$ соответственно. В свою очередь, как было показано ранее $[5,7]$, поведение спектра отражения монокристаллов и поликристаллов $\mathrm{SmS}$ стехиометрического состава хорошо описывается моделью Друде-Лоренца:

$$
\begin{aligned}
\varepsilon(v) & =\varepsilon_{1}(v)+i \varepsilon_{2}(v) \\
& =\frac{v_{p}^{2} \varepsilon_{\infty}}{-v^{2}+i v \gamma_{p}}+\frac{S_{\mathrm{ph}}}{v_{t}^{2}-v^{2}+i v \gamma_{\mathrm{ph}}}+\varepsilon_{\infty} .
\end{aligned}
$$

В этой формуле первый терм отражает вклад колебаний плазмы свободных электронов $\left(v_{p}-\right.$ плазменная частота, $\omega_{p}=2 \pi c v_{p}, \quad \gamma_{p}-$ демпфирование плазмы). Второй терм описывает вклад поперечных решеточных колебаний с помощью классического демпфированного осциллятора $\left(v_{t}-\right.$ частота колебаний, $S_{\mathrm{ph}}-$ сила осциллятора, $\gamma_{\mathrm{ph}}$ - его демпфирование). Величина $\varepsilon_{\infty}$ характеризует постоянный вклад всех остальных высокочастотных процессов. Учитывая однотипный характер спектров моно- и поликристаллов различного состава, спектры последних также можно описать с помощью

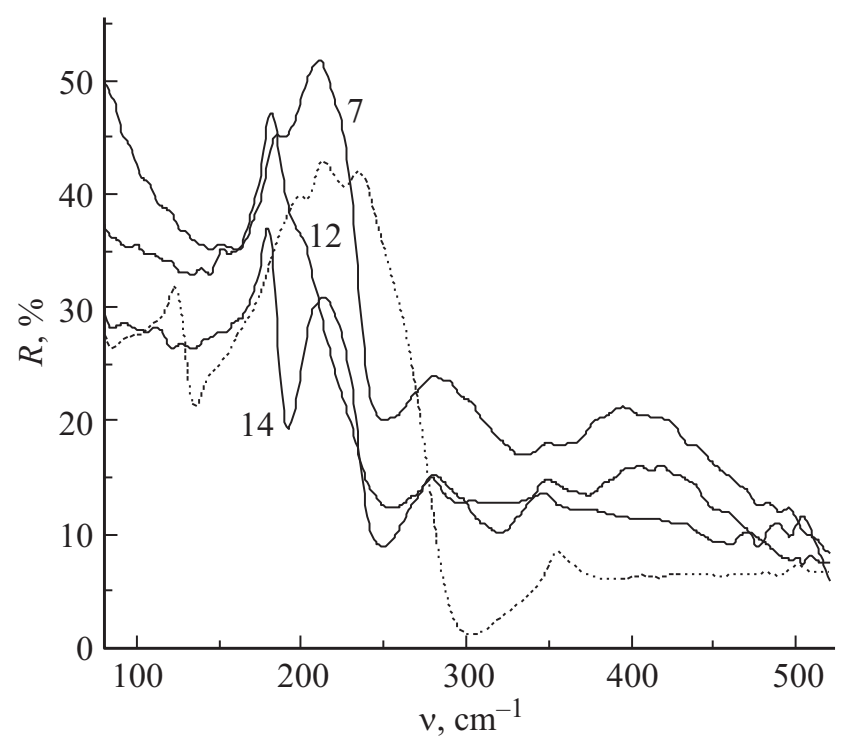

Рис. 2. Спектры отражения образцов 7, 12 и 14 (сплошные линии) и монокристалла $\mathrm{Sm}_{3} \mathrm{~S}_{4}$ (пунктирная линия).

этой модели. Вычисленное в результате проведения такой аппроксимации среднее значение частоты поперечных решеточных колебаний равно $(182.2 \pm 0.1) \mathrm{cm}^{-1}$. Остальные параметры уравнения (2) характеризуют индивидуальные особенности исследованных образцов и приведены в табл. 2.

Частотная зависимость удельной электропроводности образца может быть записана с помощью общего выражения:

$$
\sigma(\omega)=\varepsilon_{0} \omega \varepsilon_{2}(\omega),
$$

где $\varepsilon_{0}-$ абсолютная диэлектрическая проницаемость. На основании (3) из спектральных данных можно вычис-

Таблица 2. Спектральные параметры образцов

\begin{tabular}{c|l|l|r|r|r|r}
\hline $\begin{array}{c}\text { Номер } \\
\text { образца }\end{array}$ & $x$ & $\varepsilon_{\infty}$ & $\begin{array}{c}S_{\mathrm{ph}}, \\
\mathrm{cm}^{-2}\end{array}$ & $\begin{array}{c}\gamma_{\mathrm{ph}}, \\
\mathrm{cm}^{-1}\end{array}$ & $\begin{array}{c}\nu_{\mathrm{p}}, \\
\mathrm{cm}^{-1}\end{array}$ & $\begin{array}{c}\gamma_{\mathrm{p},} \\
\mathrm{cm}^{-1}\end{array}$ \\
\hline 1 & $0.00^{*}$ & 6.55 & 204000 & 7.6 & 666 & 1332 \\
2 & $0.00^{*}$ & 2.95 & 80400 & 26.2 & 908 & 2350 \\
3 & 0.00 & 3.5 & 44931 & 25.4 & 412 & 542 \\
4 & 0.02 & 2.48 & 3283 & 12.4 & 330 & 401 \\
5 & 0.02 & 1.91 & 7560 & 35.1 & 250 & 246 \\
6 & 0.04 & 2.99 & 10270 & 30.7 & 333 & 492 \\
8 & 0.05 & 3.52 & 684 & 6.8 & 264 & 293 \\
9 & 0.05 & 5.13 & 27400 & 9.3 & 449 & 418 \\
10 & 0.06 & 2.33 & 2745 & 44 & 136 & 93 \\
11 & 0.06 & 3.11 & 55000 & 34.3 & 516 & 1088 \\
13 & 0.08 & 1.3 & 5500 & 6.4 & 570 & 471 \\
15 & 0.10 & 2.08 & 1471 & 15.1 & 319 & 666 \\
16 & 0.10 & 3.32 & 27200 & 20 & 488 & 695 \\
17 & 0.15 & 3.81 & 12900 & 16 & 280 & 150 \\
18 & 0.16 & 2.71 & 614 & 6.7 & 293 & 425 \\
19 & 0.17 & 2.87 & 3605 & 11.5 & 290 & 262 \\
20 & 0.17 & 6.96 & 12900 & 5.7 & 497 & 410
\end{tabular}

Примечание. * - монокристаллический образец. 
лить статическую электропроводность как предел этого выражения при $\omega \rightarrow 0$. При условии справедливости модели Друде-Лоренца это дает выражение

$$
\sigma_{\mathrm{opt}}=\varepsilon_{\infty} v_{p}^{2} / 60 \gamma_{p},
$$

где $\varepsilon_{\infty}, v_{p}$ и $\gamma_{p}$ - параметры аппроксимации спектров, приведенные в табл. 2. Вычисленные согласно этому уравнению значения удельной электрической проводимости $\sigma_{\text {opt }}$ образцов приведены в табл. 1. Можно видеть, что для монокристалла и поликристалла $\mathrm{SmS}$ стехиометрического состава в соответствии с $[5,7] \sigma_{\text {opt }}$ хорошо совпадают с величинами $\sigma_{\mathrm{el}}$, полученными из электрических измерений. Для остальных образцов $\sigma_{\mathrm{opt}}<\sigma_{\mathrm{el}}$, несмотря на то что спектры хорошо описываются моделью Друде-Лоренца. Такие различия давно наблюдались при сравнении статической проводимости полупроводников, вычисляемой из оптических и электрических измерений, причем отклонения наблюдались как в меньшую, так и в бо́льшую стороны [12]. Устранить эти различия неоднократно пытались с помощью разнообразных методов. Суть этих попыток можно свести к двум основным подходам. Первый состоит в различных способах учета ошибки определения $v_{p}$, возникающей при ограничениях, сделанных при выводе формулы (2). Однако во всех рассмотренных случаях величина устраняемой ошибки не превышала $50 \%$ [13], в то время как различие $\sigma_{\text {opt }}$ и $\sigma_{\mathrm{el}}$ в нашем случае достигает десятикратной величины и более. Второй способ объяснения различия заключается в предположении существования приповерхностного слоя носителей заряда, влияющего на формирование отраженного сигнала, но не дающего заметного вклада в объемную электропроводность образца. На основании такого предположения в [14] была предложена поправка в формулу (1), сделанная при условии, что толщина приповерхностного слоя не превышает дебаевскую длину экранирования. Однако эта величина в случае $\mathrm{SmS}$ слишком мала $(22 \AA$ [7]) по сравнению с глубиной проникновения ИК излучения в образец $(\lambda / 2 \approx 25$ мкм $)$, что говорит о неприемлемости такого объяснения. Если допустить существование приповерхностного слоя, то для значительного влияния на спектр отраженного сигнала его толщина должна быть сравнима с глубиной проникновения ИК излучения в образец. Однако в этом случае формула для расчета, предложенная в [14], требует уточнения.

Подтверждение существования приповерхностного слоя с концентрацией свободных электронов, отличной от этой величины в объеме, можно найти также при рассмотрении их эффективных масс $m_{c}^{*}$. Оценку этих величин можно сделать с помощью известной формулы для плазменной частоты:

$$
m_{c}^{*}=\frac{4 \pi n e^{2}}{\varepsilon_{\infty} \omega_{p}^{2}} .
$$

Вычисленные в соответствии с (5) значения $m_{c}^{*}$ представлены в табл. 1. Можно видеть, что для моно- и поликристалла $\mathrm{SmS}$ стехиометрического состава эти величины меньше единицы и имеют разумные значения. Для поликристаллов других составов величины $m_{c}^{*}$ значительно превышают единицу и имеют маловероятные значения. Поскольку плазменные частоты $\omega_{p}$ определяются экспериментально, то уменьшение $m_{c}^{*}$ возможно только за счет уменьшения величины $n$. Следовательно, в поликристаллах $\mathrm{Sm}_{1+x} \mathrm{~S}(x=0-0.17)$ концентрации электронов $n$ в приповерхностном слое действительно отличаются в менышую сторону от этих же величин в объеме. Тем самым становятся понятными и меньшие значения оптических электропроводностей. Таким образом, на основании измерения спектров отражения поликристаллических образцов можно сделать вывод о существовании на их поверхности особого слоя, электрические характеристики которого значительно отличаются от характеристик в объеме.

Ранее в [15] было показано, что часть донорных уровней в $\mathrm{SmS}$ заблокированы из-за образования оксисульфида самария, $\mathrm{Sm}_{2} \mathrm{O}_{2} \mathrm{~S}$. При этом большая часть оксисульфида образуется в приповерхностных областях образца. Однако в настоящей работе такие образцы имели другие спектры отражения (см. рис. 1 и 2) и были заранее исключены из рассмотрения. Поэтому при рассмотрении образцов, спектры которых представлены на рис. 1 , речь идет о приповерхностном слое образцов с составом $\mathrm{Sm}_{1+x} \mathrm{~S}$.

Еще одной интересной особенностью поликристаллов $\mathrm{SmS}$ является значительное уменьшение в них силы осциллятора фононных колебаний $S_{\mathrm{ph}}$ (см. табл. 2). Для понимания природы этого явления на основании параметров аппроксимации спектров был рассчитан микроскопический эффективный заряд иона $e_{s}^{*}$ (табл. 1), определяющий ионность химической связи (формула Сигети [16]), который в наших обозначениях запишется в виде

$$
e_{s}^{*}=\frac{1}{e}\left(\pi S_{\mathrm{ph}}\right)^{1 / 2}\left(\frac{M}{N}\right)^{1 / 2} \frac{3 c}{\varepsilon_{\infty}+2},
$$

где $e$ - заряд электрона, $c$ - скорость света, $M=M_{\mathrm{Sm}} M_{\mathrm{S}} /\left(M_{\mathrm{Sm}}+M_{\mathrm{S}}\right)-$ приведенная масса ионов, $N$ - число пар ионов в единице объема.

Для монокристаллов величины эффективного заряда имеют наибольшие значения, близки к единице и хорошо совпадают с аналогичными величинами, вычисленными для монохалькогенидов европия [17]. Для поликристаллических образцов эти величины имеют меньшие значения, зависящие от концентрации электронов (рис. 3). Эту зависимость можно описать следующим соотношением:

$$
e_{s}^{*}=0.66-0.015 n,
$$

где $n-$ концентрация электронов в единицах $10^{18} \mathrm{~cm}^{-3}$.

Наблюдаемое уменьшение ионности связи в поликристаллах $\mathrm{Sm}_{1+x} \mathrm{~S}$ на самом деле является мнимым и объясняется увеличением экранирования кулоновского взаимодействия между ионами при увеличении концентрации электронов проводимости. В [18] было показано, 


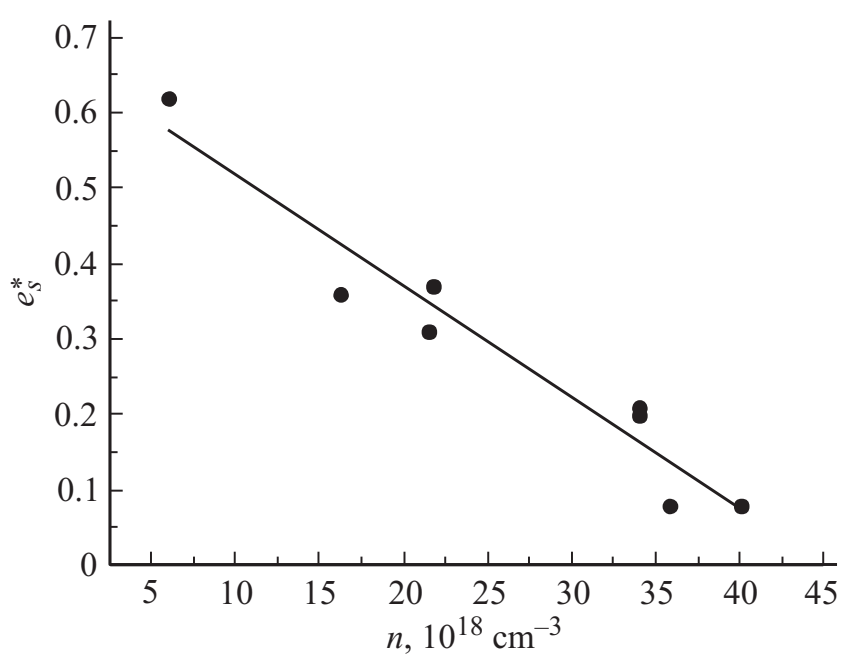

Рис. 3. Зависимость заряда Сигети от концентрации электронов в исследованных образцах.

что величина ОКР определяет степень дефектности поликристаллов $\mathrm{Sm}_{1+x} \mathrm{~S}$ и, следовательно, концентрацию донорных уровней $N_{i}$, поскольку между этими величинами существует однозначная связь. Из табл. 1 видно, что особенно существенное уменьшение величины ОКР происходит при переходе от моно- к поликристаллам. Это приводит к возрастанию концентрации ионов самария, находящихся вне регулярных узлов кристаллической решетки $\mathrm{SmS}$. По мере увеличения $x$ концентрация донорных уровней $N_{i}$ и соответственно концентрация электронов возрастают еще больше, что и приводит к увеличению экранирования.

\section{4. Заключение}

По результатам работы можно сделать следующие выводы.

- В моно- и поликристаллических образцах $\mathrm{SmS}$ стехиометрического состава удельную электропроводность можно определять с точностью $\sim 10 \%$ из измерений спектров отражения.

- Для поликристаллов $\mathrm{Sm}_{1+x} \mathrm{~S}(0<x \leq 0.17)$ наблюдаются значительные отклонения электропроводности, определенной оптическим методом, в меньшую сторону по сравнению с определенной электрическим методом. Это происходит вследствие наличия приповерхностного слоя образца с меньшей электропроводностью по сравнению с остальным объемом.

- Концентрация электронов проводимости в поликристаллических образцах $\mathrm{Sm}_{1+x} \mathrm{~S}$ увеличивается по мере уменьшения величины ОКР и увеличения концентрации донорных примесей. Для оценки такого увеличения может служить величина микроскопического эффективного заряда, определяющая степень ионности связи $\mathrm{Sm}-\mathrm{S}$ и вычисляемая из спектров отражения.

\section{Список литературы}

[1] В.В. Каминский, А.А. Молодых, С.М. Соловьев, А.А. Виноградов, Н.М. Володин. Патент РФ на изобретение № 2564698. Приоритет от 28.02.2014.

[2] В.М. Егоров, В.В. Каминский, М.М. Казанин, С.М. Соловьев, А.В. Голубков. Письма ЖТФ, 41, 50 (2015).

[3] С.А. Казаков, В.В. Каминский, С.М. Соловьев, Н.В. Шаренкова. Научное приборостроение, 25, 116 (2015).

[4] А.В. Голубков, Е.В. Гончарова, В.П. Жузе, Г.М. Ложнов, В.М. Сергеева, И.А. Смирнов. Физические свойства халькогенидов редкоземельных элементов (Л., Наука, 1973).

[5] Ю.В. Улашкевич, В.В. Каминский, М.М. Казанин. ФТТ, 54, 2066 (2012).

[6] Г.П. Скорняков, В.Л. Константинов. В сб.: Физика и химия Р3-полупроводников (Свердловск, 1977) с. 20.

[7] V. Zelezny, J. Petzelt, V.V. Kaminski, M.V. Romanova, A.V. Golubkov. Solid State Commun., 72, 43 (1089).

[8] Ю.В. Улашкевич, В.В. Каминский, А.В. Голубков. ФТП, 43, 323 (2009).

[9] А.В. Голубков, Т.Б. Жукова, В.М. Сергеева. Неорг. матер., 2, 77 (1966).

[10] Н.В. Шаренкова, В.В. Каминский, А.В. Голубков, Л.Н. Васильев, Г.А. Каменская. ФТТ, 4, 598 (2005).

[11] В.М. Сергеева и др. Неорг. матер., 8, 2114 (1972).

[12] G. Navascues, F. Flores. Solid State Commun., 9. 1261 (1971).

[13] В.Д. Кулаковский, В.Д. Егоров. ФТТ, 15, 2053 (1973).

[14] F. Flores, F. Garsia-Voliner, G. Navascues. Surf. Sci., 24, 61 (1971).

[15] Л.Н. Васильев, В.В. Каминский, М.В. Романова, Н.В. Шаренкова, А.В. Голубков. ФТТ, 10, 1777 (2006).

[16] B. Szigeti. Trans. Farad. Soc., 45, 155 (1949).

[17] J.D. Axe. J. Phys. Chem. Solids, 30, 1403 (1969).

[18] Н.В. Шаренкова, В.В. Каминский, М.В. Романова, Л.Н. Васильев, Г.А. Каменская. ФТТ, 50, 1158 (2008).

Редактор Л.В. Шаронова

\section{Investigation of long-wavelength infrared reflection spectra of SmS mono- and polycrystals in the region of homogeneity}

Y.V. Ulashkevich ${ }^{1}$, V.V. Kaminskiy ${ }^{2}$, M.V. Romanova ${ }^{2}$, N.V. Sharenkova ${ }^{2}$

${ }^{1}$ State Institute of Technology,

190013 St. Petersburg, Russia

2 loffe Institute,

194021 St. Petersburg, Russia

Abstract The reflection spectra of $\mathrm{Sm}_{1+x} \mathrm{~S}$ samples $(x=0-0.17)$ in the far and middle IR regions and their electrical and structural parameters at the temperature of $T=300 \mathrm{~K}$ were recorded and analyzed. It is shown, that the degree of ionicity of the bond in SmS decreases with decreasing of the size of $X$-ray coherent scattering region, increasing of the concentration of donor impurities, and as a results, with increasing of the concentration of conduction electrons. In mono- and polycrystals of $\mathrm{SmS}$ of stoichiometric composition, the electrical conductivity can be determined from the IR reflection spectra with the accuracy of $10 \%$. In the case of deviation from stoichiometry, increased defect concentration in the samples does not allow to determine the electrical conductivity. 